\title{
Benefits of being bilingual? The relationship between pupils' perceptions of teachers' appreciation of their home language and executive functioning
}

International Journal of Bilingualism 2016, Vol. 20(6) 700-713 (C) The Author(s) 2015 Reprints and permissions: sagepub.co.uk/journalsPermissions.nav DOI: $10.1177 / 1367006915586470$ ljb.sagepub.com

@SAGE

\section{Claire Goriot}

Centre for Language Studies \& Behavioural Science Institute, Radboud University, The Netherlands

\section{Eddie Denessen, Joep Bakker and Mienke Droop}

Behavioural Science Institute, Radboud University, The Netherlands

\begin{abstract}
Aims: We aimed to investigate whether bilingual pupil's perceptions of teachers' appreciation of their home language were of influence on bilingual cognitive advantages.

Design: We examined whether Dutch bilingual primary school pupils who speak either German or Turkish at home differed in perceptions of their teacher's appreciation of their HL, and whether these differences could explain differences between the two groups in executive functioning.

Data and analysis: Executive functioning was measured through computer tasks, and perceived home language appreciation through orally administered questionnaires. The relationship between the two was assessed with regression analyses.

Findings: German-Dutch pupils perceived there to be more appreciation of their home language from their teacher than Turkish-Dutch pupils. This difference did partly explain differences in executive functioning. Besides, we replicated bilingual advantages in nonverbal working memory and switching, but not in verbal working memory or inhibition.

Originality and significance: This study demonstrates that bilingual advantages cannot be dissociated from the influence of the sociolinguistic context of the classroom. Thereby, it stresses the importance of culturally responsive teaching.
\end{abstract}

\section{Keywords}

Bilingualism, executive functioning, sociolinguistic context, culturally responsive teaching, primary school 


\section{Introduction}

Just as in other countries, many inhabitants of the Netherlands speak more than one language. Most frequently, these are languages from ethnic minority groups, like Turkish and Arabic, and languages from other European countries, mostly English and German. As previous research has revealed, bilingualism seems to foster advantages over monolingualism, especially in the cognitive domain (Adesope, Lavin, Thompson, \& Ungerleider, 2010; Bialystok, 2009). A question is whether these advantages are equal for all bilinguals, or whether they differ according to the sociolinguistic context in which bilinguals are embedded. In this exploratory study, we focus specifically on bilingual children's perceptions of their teachers' appreciation of their home language (HL), to explain how contextual influences may affect cognitive advantages of bilingualism.

\section{Cognitive advantages of being bilingual}

The cognitive advantages of bilingualism seem to be especially prominent in executive functioning (EF), an umbrella term for multiple inter-related processes which are together responsible for goaldirected behaviour, and reflection on it (St Clair-Thompson \& Gathercole, 2006). EF is, among other things, important for academic achievement (Blair \& Peters Razza, 2007; St Clair-Thompson \& Gathercole, 2006), and adult health and wealth (Moffitt et al., 2011). Since EF development may positively be influenced by the socioeconomic environment in which children grow up (Hackman \& Farah, 2009), the socioeconomic status (SES) cannot be ignored in research concerning EF. It is often assumed that EF consists of three key processes (Garon, Bryson, \& Smith, 2008; Miyake, Friedman, Emerson, Witzki, Howerter, \& Wager, 2000): (1) inhibition, can be described as being able to control one's attention to override preliminary responses and to act more deliberately, being able to resist temptations; (2) switching (or shifting), the ability to flexibly adjust to different rules or demands and the capability to rapidly adapt to changed circumstances; and (3) working memory (WM), the process of manipulating information that is held in mind, which can be subdivided into verbal and nonverbal WM (Diamond, 2013).

EF development takes place during childhood, with infants being able to hold information in mind. Mentally manipulating this information takes much longer to develop, just as switching and inhibition. It is not until adolescence that all processes are fully matured (Diamond, 2013). The development of EF can be improved by computerized or educational programs as well as by bilingual input (Diamond, 2013). In this study, we investigate the effects on EF development of an educational context in which bilingualism is more or less appreciated. Bilingual advantages in EF have been studied extensively. Although some studies (see, for example, Antón et al., 2014) fail to demonstrate advantages, a fairly large amount of research has demonstrated advantages for bilingual children in switching and inhibition (see for example, Bialystok \& Viswanathan, 2009) and in WM (see, for example, Morales, Calvo, \& Bialystok, 2013).

Although there is still some discussion about the explanation of these advantages, a general assumption is that bilinguals have to mentally control two languages simultaneously (Green, 1998). Words that show phonological or semantic overlap are activated in parallel and compete with each other for selection. While monolinguals only have to inhibit this competition in one language, bilinguals have to inhibit overlapping words in two languages. Because they have to deal with linguistic competition to a broader extent, bilinguals would show advantages in linguistic control which would generalize to nonlinguistic control processes like EF (Green, 1998). Advantages appear to occur regardless of whether two languages are related or not (Barac \& Bialystok, 2012). These advantages are especially likely to develop when the second language (L2) is acquired at a young age and when both languages are used regularly (Carlson \& Meltzoff, 2008) and alternately (Green, 1998). L2 proficiency seems to be less important, since advantages are also found for bilingual populations who are less proficient in one of their languages (Bialystok \& Martin, 2004). 
Despite the fact that advantages for bilingual children in the key processes of EF have been well established (Adesope et al., 2011; Bialystok, 2009), a core question is whether these advantages are equal for all bilingual children. Besides the age of onset and balance in language use (Luk, De Sa, \& Bialystok, 2011; Soveri, Rodriguez-Fornells, \& Laine, 2011), advantages might be dependent on other, more environmental aspects, such as the sociolinguistic context bilinguals are immersed in. The way in which languages are valued depends on the sociolinguistic context, and thus which status is attributed to a language (Appel \& Muysken, 2005). The differential value of some languages over others has been found to be related to the social status that is attributed to groups in society. Languages that ethnic minority groups speak are likely to have a relatively low status, because these languages can be associated with groups that, on average, have low levels of education, low paid jobs, and higher levels of unemployment (Van Gaalen \& De Vos, 2011).

The sociolinguistic context, and therefore the status of languages, is affected by governmental policies regarding languages (Appel \& Muysken, 2005). At present, the Dutch government shows an ambivalent attitude towards bilingualism. On the one hand, it encourages "bilingual primary school education", a type of education in which pupils receive extra hours of foreign language education. In the Netherlands, this foreign language is always another European language, like English (Kuiken \& Van der Linden, 2014). On the other hand, the government discourages the use of some other languages, especially languages with a low status. Ethnic minority students are often urged to speak Dutch instead of their HL, particularly because speaking the HL is considered to hinder integration in society (Agirdag, Jordens, \& Van Houtte, 2014; Kuiken \& Van der Linden, 2014). Furthermore, in the context of policies aimed at reducing educational inequalities, the Dutch government assumes that speaking the HL will impair the use of the majority language, and therefore will increase a language delay in Dutch (Leseman, 2000). This assumption, however, does not find a solid basis in the research literature (Atwill, Blanchard, Christie, Gorin, \& García, 2010; Eisenchlas, Schalley, \& Guillemin, 2013). Despite this, educational provisions like school programs to promote the language of ethnic minority groups have been discarded (Extra and Yağmur, 2004; Kuiken \& Van der Linden, 2014).

Regardless of the stance of the Dutch government towards the use of ethnic minority languages in schools, teachers may vary in their attitudes towards the use of different languages at school. They may contribute to the level of bilingualism in their classroom by promoting the use of pupils' $\mathrm{HL}$ at school. As a result, they may add to the cognitive benefits of bilingualism by providing an encouraging sociolinguistic context.

\section{Teachers' cultural responsiveness}

In a classroom, teachers may adapt their communication and instruction practices to culturalspecific particularities (Gay, 2002), of which the HL of the child is part. These so-called culturally responsive teachers may, for example, make use of the child's HL during their lessons (Chun \& Dickson, 2011), or praise children for being bilingual (Lee \& Oxelson, 2011). They intend to diminish the gap between children's home and school culture (Brown, 2007), and teach them more effectively. Therefore, learning experiences would be more personally meaningful and valuable for ethnically diverse pupils in classrooms with culturally responsive teachers (Gay, 2002). The teacher's cultural responsiveness relates to the extent to which children perceive that their HL is appreciated. Children taught by teachers who pay more attention to their HL may perceive that their HL is appreciated more, and therefore is of a relatively higher status, than children taught by teachers who are less sensitive towards their HL.

Some teachers seem to be less culturally responsive than others. Gkaintartzi and Tsokalidou (2011), for example, interviewed teachers in Greek primary schools about their attitudes towards bilingual education for Albanian minority pupils. They found that some teachers showed awareness of the importance of bilingualism, while others rejected the use of Albanian in the classroom. 
By discarding the pupils' HL, and by imposing the use of the majority language, teachers may reflect that a low status is attributed to pupils' HL.

Agirdag (2010) showed that students are aware of their teachers' appreciation of their HL. He interviewed Turkish-Dutch bilingual secondary school students, who indicated that they felt that their teachers appreciated ethnic minority languages less than western languages. They were, for example, allowed to read the newspaper in English, but prohibited from reading one in Turkish. Although research has shown that children perceive differential appreciations of different languages, not much research has been done on the effects of these perceptions on children's cognition.

From the abovementioned research it appears that teachers may affect the status of the different languages of bilingual pupils in the classroom by the extent to which they value and/or tolerate different HLs. They may even negatively influence the extent to which children can use their HL, by urging children to use the majority language. Thus, they may restrict two important requirements to develop advantages in EF, namely usage of and switching between two languages (Carlson \& Meltzoff, 2008; Green, 1998).

\section{The present study}

We focus on bilingual children who are, besides Dutch, proficient in one of the two most common second languages in the Netherlands, namely Turkish or German. In Dutch society, German can be assumed to have a higher status than Turkish, because the Turkish-Dutch population in general has a lower socioeconomic status than the German-Dutch population. The research question is to what extent bilingual pupils with these HLs experience differences in the appreciation of their HL by their teachers, and whether these experiences influence the key EF processes: inhibition, switching, and working memory.

The first aim is to re-demonstrate previously established bilingual advantages in EF, and second, to identify whether bilingual pupils who are proficient in different HLs show comparable levels of EF. Third, we investigate whether bilingual pupils' EF is influenced by their perceptions of their teachers' appreciation of the HL. It is expected that bilingual children who experience their teacher as discouraging the use of their HL will show less well developed EF.

\section{Method}

\section{Participants}

The participants were 71 children and their parents, living all over the Netherlands (see Table 1). Recruitment took place by advertisements on websites for German and Turkish people in the Netherlands, and by addressing two schools. To avoid dependency of the data, one pupil per teacher was tested, to prevent children in the sample from sharing the same classroom context.

Through orally administered questionnaires, parents confirmed that children were only proficient in Dutch and either German or Turkish and used both languages regularly (see Table 1). Information about parents' education level was obtained as a measure of SES. For two TurkishDutch children, parents did not provide this information. Although monolingual Dutch-speaking children reported some use of English, they could not be considered proficient in this language. SES was not equally divided among groups $\left(\chi^{2}(4)=20.466, p<.001\right)$; Turkish-Dutch children in this sample appeared to have lower socioeconomic backgrounds than the other two groups. No significant group differences in age existed $(F(2,68)=2.25, p=.113)$. 
Table I. Descriptive statistics per language group.

\begin{tabular}{|c|c|c|c|c|}
\hline & & Dutch & Dutch-German & Dutch-Turkish \\
\hline \multirow[t]{2}{*}{ Gender } & Boys $(N)$ & 13 & 17 & 9 \\
\hline & Girls (N) & 10 & 8 & 14 \\
\hline Age & $M(S D)$ & $8.99(0.68)$ & $9.49(0.75)$ & $9.11(1.10)$ \\
\hline \multirow[t]{3}{*}{ SES } & Low $(N)$ & 0 & 0 & 3 \\
\hline & $\operatorname{Medium}(N)$ & 4 & 0 & 8 \\
\hline & High $(N)$ & 19 & 25 & 10 \\
\hline$\%$ use of Dutch (child rating) & $M(S D)$ & 91.78 (3.32) & $62.30(14.96)$ & $53.31(12.44)$ \\
\hline \% use of $\mathrm{HL}$ (child rating) & $M(S D)$ & - & $34.26(14.03)$ & $43.26(12.60)$ \\
\hline$\%$ use of Dutch (parent rating) & $M(S D)$ & $89.69(3.70)$ & $57.17(12.5 \mathrm{I})$ & $54.38(15.38)$ \\
\hline$\%$ use of $\mathrm{HL}$ (parent rating) & $M(S D)$ & - & $40.75(15.38)$ & $43.60(14.54)$ \\
\hline \multirow[t]{3}{*}{ Nonverbal WM } & $N$ & 23 & 25 & 23 \\
\hline & $M(S D)$ & $4.65(0.93)$ & $5.32(0.90)$ & $5.17(1.30)$ \\
\hline & Range & $3-6$ & $4-7$ & $3-9$ \\
\hline \multirow[t]{3}{*}{ Verbal WM } & $N$ & 23 & 25 & 23 \\
\hline & $M(S D)$ & $4.00(1.17)$ & $4.96(1.40)$ & $3.87(1.01)$ \\
\hline & Range & $2-7$ & $3-7$ & $2-5$ \\
\hline \multirow[t]{3}{*}{ Switching } & $N$ & 23 & 25 & 23 \\
\hline & $M(S D)$ & $77.53(68.44)$ & $29.02(66.25)$ & $55.65(62.31)$ \\
\hline & Range & $-49.32-231.83$ & $-113.79-179.06$ & $-58.32-180.06$ \\
\hline \multirow[t]{3}{*}{ Inhibition } & $N$ & 22 & 25 & 17 \\
\hline & $M(S D)$ & $150.07(131.64)$ & $200.09(128.83)$ & $151.39(137.00)$ \\
\hline & Range & $-74.08-451.19$ & $-81.7 \mid-453.81$ & $-|09.7|-495.27$ \\
\hline HL tolerance & $M(S D)$ & - & $1.86(0.25)$ & $\mathrm{I} .40(0.33)$ \\
\hline $\mathrm{HL}$ value & $M(S D)$ & - & $1.62(0.32)$ & $1.38(0.27)$ \\
\hline Stimulation of Dutch & $M(S D)$ & - & $1.33(0.26)$ & I.5I (0.28) \\
\hline \multirow[t]{2}{*}{ Raven's test } & $M(S D)$ & $29.61(3.13)$ & $32.44(2.47)$ & 27.91 (4.09) \\
\hline & Range & $24-34$ & $25-36$ & $18-35$ \\
\hline
\end{tabular}

\section{Measures}

\section{Children's bilingualism}

Language use. In an orally administered questionnaire, parents were questioned on the age at which children learned their language(s), and on children's language use during diverse activities, 
like watching television. Children were also asked about their language use during diverse activities, like when playing with friends. Parents and children could indicate whether children do these activities never, sometimes, half of the time, often, or always in Dutch. Bilingual children and their parents also had to indicate how often children use their HL. Reliability of the questionnaires was high $(\alpha=.75$ for parents' ratings of HL use, $\alpha=.85$ for parents' ratings of use of Dutch, $\alpha=.78$ for children's ratings of HL use, and $\alpha=.88$ for children's ratings of use of Dutch; Cronbach, 1951). Percentage scores were computed, which indicated how often children used Dutch or their HL (see Table 1).

\section{Executive functioning}

Inhibition. The Flanker Fish task (Diamond, Barnett, Thomas, \& Munro, 2007) consisted of three blocks involving blue, pink, and mixed trials, respectively. Children saw five fish on a row, appearing in different combinations: either the stimulus or all fish could be presented, and distracter fish could be pointing in the same or opposite direction from the stimulus fish, or downward. Children were instructed to pay attention to the stimulus fish, which was the middle fish in case of the blue and the outer fish in case of the pink ones. Children had to press the corresponding key on the keyboard to indicate whether the fish were pointing left or right. Response times were restricted to $1500 \mathrm{~ms}$. The first two blocks were preceded by instructions and four practice trials, followed by 16 experimental trials. The third block, consisting of 44 trials, was only preceded by instructions.

Misses, incorrect responses, responses below $200 \mathrm{~ms}$ or above $1500 \mathrm{~ms}$ were deleted $(19.3 \%$ in total). Subsequently, inhibition scores were computed as the difference between the mixed block and the pink fish block. Data for seven participants (one monolingual and six Turkish-Dutch children) were missing or removed due to technical errors or because performance in terms of reactions times or accuracy was predominantly erroneous.

Switching. In a slightly modified version of the Faces task (Bialystok \& Viswanathan, 2009), children were presented with a schematic face, flanked by two boxes. Subsequently, the eyes of the face turned either red or green, after which the face disappeared leaving only the two boxes. Then, an asterisk flashed in either the left or the right box. Children had $1500 \mathrm{~ms}$ to react, and were instructed to press the marked key on the keyboard that was on the same side of the asterisk if the eyes were green, and on the opposite side of the asterisk if the eyes were red.

The task was divided into four single colour and four mixed colour blocks of 24 trials each. Half of the blocks consisted of straight gaze trials, and the other half of shift gaze trials, in which the eyes were looking to the left or the right instead of straight ahead. Prior to the straight and shift eyes blocks, participants received instructions and a practice block of eight trials. The same procedure as for the Flanker Fish task was used to remove incorrect and deviating responses (23.6\% in total). Switching was assessed by comparing mean reaction times of the mixed and single colour blocks.

Nonverbal working memory. In the Corsi Block-tapping task (Vandierendonck, Kemps, Fatame, \& Szmalec, 2004), children were presented with nine blue blocks irregularly positioned on the black computer screen. The blocks changed shortly into yellow at a rate of one block per second. Children were instructed to click on the blocks in the exact same order with which they changed colour. The task began with a sequence of two blocks and increased by one after two correct trials, with a maximum of nine. Testing ended when two trials of the same sequence were remembered incorrectly. The score was determined by the span length of the final trial that the child performed correctly.

Verbal working memory. In the Backward Digit Span (Kort et al., 2005), a string of numbers was orally presented to the child, who had to repeat it in reverse order. The task began with a sequence 
of two numbers that increased by one after two correct trials. Testing ended when two trials of the same sequence were remembered incorrectly. The total score was calculated as the number of correctly recalled sequences, which could range between 0 and 18 .

\section{Sociolinguistic context measures}

Perceptions of teachers' appreciation of the HL. A questionnaire was developed, consisting of 11 statements that were partly based on existing questionnaires (Chun and Dickson, 2010; Lee \& Oxelson, 2006). The statements were adjusted for use with Dutch primary school pupils. Children could indicate whether each statement was true or false, to prevent them from choosing a neutral category. The mean score could range between 1, which indicated that children had the feeling that the teacher did not appreciate the HL at all, and 2, which indicated that children had the feeling that the teacher appreciated the HL very much.

We conducted an exploratory factor analysis (with varimax rotation) to assess which aspects of HL appreciation could be distinguished. The scree plot pointed to the existence of three dimensions: HL tolerance, HL value, and stimulation of Dutch. One item that did not fit any of the factors was removed from the analyses. Final factor loadings are presented in Table 2. Together, these factors represent $58.1 \%$ of the observed item variance. 'HL tolerance' consisted of four items (Cronbach's alpha coefficient was .79). 'HL value' and 'stimulation of Dutch' both consisted of three items (Cronbach's alpha coefficients were .43 and .56, respectively). For each scale, a score was computed by averaging the scores on each of the items. 'Stimulation of Dutch' was negatively and statistically significant related to 'HL tolerance' $(r=-.348, p=0.015)$, but not to 'HL value' $(r=-.059, p>.05)$. The latter two dimensions were weakly positively, but not statistically significantly, related $(r=.269, p>.05)$.

Intelligence. Raw scores on Raven's Coloured Progressive Matrices (Raven, Court, \& Raven, 1990), a widely used measure (Bialystok \& Martin, 2004; Blair \& Peters-Razza, 2007), were assessed to study how differences between groups may be related to differences in fluid nonverbal intelligence. Children were presented with 36 patterns with a missing part, which they had to choose out of six alternatives. The score was computed as the total number of correct items.

Procedure. Children were tested individually in a quiet room in their home environment $(N=62)$, or, when recruited through schools, at school $(N=9)$. Tasks were presented in a fixed order. The Flanker Fish task was programmed in Presentation (Neurobehavioral Systems, Inc.), the Faces and the Corsi Block-tapping tasks in Inquisit (Millisecond Software). Children started with the Flanker Fish task, followed by the Faces task, the Corsi Block-tapping task, the Backward Digit Span, and the Raven's test. An orally administered questionnaire about children's language use and their perceived teacher appreciation of their HL concluded the session. Monolingual children were only questioned about their language use. Children were tested in Dutch in one session of approximately one hour and received a gift for their participation. Parents were asked to answer questions about the language use of their child. Upon request, they could get a questionnaire that was translated in the HL.

\section{Analysis}

Analyses were conducted in three steps. First, monolingual and bilingual children's performances on EF measures were compared by means of ANOVAs. More specifically, two contrasts were tested: the first one to compare the performance of bilinguals and monolinguals, the second one to compare performances of the two bilingual groups. To indicate the effect sizes, $\eta^{2}$ were 
Table 2. Factor loadings on perceptions of teachers' appreciation of the HL: Questionnaire $(N=48)$.

\begin{tabular}{|c|c|c|c|}
\hline & \multicolumn{3}{|l|}{ Factor } \\
\hline & HL tolerance & $\mathrm{HL}$ value & $\begin{array}{l}\text { Stimulation } \\
\text { of Dutch }\end{array}$ \\
\hline $\begin{array}{l}\text { 3. I have the feeling that the teacher does not approve it } \\
\text { when I talk Turkish.*** }\end{array}$ & .36 & -.16 & .34 \\
\hline $\begin{array}{l}\text { 4. The teacher does not allow me to talk Turkish in the } \\
\text { classroom with other children.** }\end{array}$ & .77 & -.31 & .04 \\
\hline $\begin{array}{l}\text { 7. I have the feeling that it does not matter to the } \\
\text { teacher whether I speak Turkish or Dutch. }\end{array}$ & -.60 & .01 & -.04 \\
\hline $\begin{array}{l}\text { 8. The teacher does not allow me to speak Turkish in } \\
\text { the playground. ** }\end{array}$ & .84 & -.23 & .36 \\
\hline $\begin{array}{l}\text { 5. I have the feeling that the teacher appreciates that I } \\
\text { also speak another language than Dutch. }\end{array}$ & -.14 & .37 & .03 \\
\hline $\begin{array}{l}\text { 6. The teacher sometimes compliments me because I } \\
\text { can also speak Turkish. }\end{array}$ & .09 & .91 & -.06 \\
\hline $\begin{array}{l}\text { II. The teacher sometimes asks me how you say } \\
\text { something in Turkish. }\end{array}$ & -.12 & .27 & .02 \\
\hline $\begin{array}{l}\text { I. The teacher has spoken in Turkish to me or to other } \\
\text { children who speak Turkish.*** }\end{array}$ & -.06 & .22 & -.30 \\
\hline $\begin{array}{l}\text { 9. I have the feeling that the teacher appreciates it more } \\
\text { when I speak Dutch at home instead of Turkish. }\end{array}$ & .07 & .00 & .61 \\
\hline $\begin{array}{l}\text { 10. I have the feeling that the teacher finds it important } \\
\text { that I always speak Dutch. }\end{array}$ & .10 & .11 & .72 \\
\hline Explained variance in percentages & 30.07 & 15.42 & 12.55 \\
\hline
\end{tabular}

*For German bilingual pupils, 'Turkish' was replaced by ‘German'.

**Before calculating scale scores, the scores on this item were recoded, so that a higher score meant more perceived tolerance.

***B Before calculating scale scores, the scores on this item were recoded, so that a higher score meant more perceived stimulation of Dutch.

calculated (Cohen, 1988). Second, we conducted independent samples $t$-tests, to compare the two bilingual groups' perceptions of teacher's appreciation of the HL. Cohen's $d$ (Cohen, 1988) was used as a measure of the effect size. Third, mediation analyses were conducted in order to assess whether perceived appreciation of the HL could explain differences between the two bilingual groups in EF performance. In other words, it was assessed whether perceived appreciation of the HL mediated the relationship between the child's HL and EF. Therefore, a categorical variable was computed, providing information about whether the child's HL was German or Turkish. Following the steps described by Baron and Kenny (1986), linear regression analyses were performed to test the expected mediation effect. The first regression analysis assessed the relationship between the HL and EF outcomes. The second one assessed the relationship between the HL and perceived appreciation of the HL. The third analysis examined whether perceived appreciation of the HL influenced EF processes, and by taking into account the HL in the regression analysis as well, whether this could explain the relationship between the HL and EF processes. We calculated $R^{2}$ (Cohen, 1992) to assess the size of these effects. Sobel tests (Sobel, 1982) were conducted to investigate whether the mediation effect was statistically significant. The set level of significance was .05. 


\section{Results}

\section{Executive functions}

As the descriptive statistics show (Table 1), overall the German-Dutch bilinguals performed better than the other two groups on all EF-measures, except for the inhibition task. They remembered longer sequences on the Corsi Block-tapping task and the Digit Span, and were faster on the Faces task. The contrast tests revealed that there were significant differences between bilingual and monolingual children in nonverbal WM $(t(68)=-2.22, p=.030)$, and in switching $(t(68)=2.11, p$ $=.038)$ : bilinguals performed better on these tasks than monolinguals. Both effects were small $\left(\eta^{2}\right.$ $=.07$, and $\eta^{2}=.09$, respectively). No significant differences were observed between the bilingual and monolingual groups in inhibition $(t(68)=-0.19, p=.466)$, or in verbal WM $(t(68)=-1.35, p=$ $.181)$. The two bilingual groups differed significantly in verbal $\mathrm{WM}(t(68)=3.12, p=.003)$ : German bilinguals scored higher than Turkish bilinguals. The effect was medium $\left(\eta^{2}=.15\right)$.

\section{Intelligence}

Significant differences between the three groups were observed in the scores on the Raven's test $(F(2,68)=11.76, p<.001$; see Table 1$)$. To assess whether these differences influenced EF, regression analyses with the Raven's test raw score as independent and EF scores as dependent variables were conducted. Intelligence scores had a significantly positive effect on the scores of nonverbal $\mathrm{WM}(b=0.10, S E=0.03, p=.003)$ and verbal WM $(b=0.14, S E=0.04, p<.001)$. These effects were small $\left(R^{2}=.12\right)$ and medium $\left(R^{2}=.17\right)$, respectively. No significant relationships with either switching or inhibition were found $(p>.05)$. Intelligence was not included in the analyses, because of a power issue due to the small sample size.

\section{SES}

Differences in EF may be confounded with differences in SES. Since statistically controlling for SES was impossible due to the small groups, post-hoc analyses were conducted. Since all GermanDutch children's parents had a high SES, no analyses could be conducted for these children. For the two other groups, SES did not significantly influence children's EF $(p>.05)$. SES was not included as a control variable in the regression analyses since it would reduce the statistical power of the analysis.

\section{Perceived teacher appreciation of bilingualism}

The descriptive statistics of the orally administered questionnaires, displayed in Table 1, show that German-Dutch pupils compared to Dutch-Turkish pupils experienced more tolerance towards and value of their HL, and were less stimulated to use Dutch. Independent samples $t$-tests revealed that there were indeed significant differences between the two groups in the extent to which their teacher tolerated their $\operatorname{HL}(t(46)=5.41, p<.001, d=1.60)$, valued their $\operatorname{HL}(t(46)=2.73, p=.009$, $d=.81)$, and stimulated them to use Dutch $(t(46)=-2.32, p=.025, d=-0.68)$. The first two effects were large; the latter one was of medium size.

We then addressed the question of whether bilingual pupils' perception of their teachers' appreciation of their HL would influence their EF performances. Regression analyses showed that perceived HL tolerance was significantly associated with both nonverbal WM $(b=1.32, S E=0.45$, $p=.005)$, and verbal WM $(b=1.23, S E=0.57, p=.035)$. Both effects were medium $\left(R^{2}=.18\right.$ and 
Table 3. Linear regression analyses to assess mediating effects of perceived $\mathrm{HL}$ appreciation on the relationship between $\mathrm{HL}$ and $\mathrm{EF}$.

\begin{tabular}{|c|c|c|c|c|c|c|c|c|c|c|c|c|}
\hline & \multicolumn{2}{|c|}{ Effect of $\mathrm{HL}$} & \multicolumn{2}{|c|}{$\begin{array}{l}\text { Partial } \\
\text { effect of } \mathrm{HL} \\
\text { tolerance }\end{array}$} & \multicolumn{2}{|c|}{$\begin{array}{l}\text { Partial effect } \\
\text { of } \mathrm{HL} \text { value }\end{array}$} & \multicolumn{2}{|c|}{$\begin{array}{l}\text { Partial effect } \\
\text { of stimulation } \\
\text { of Dutch }\end{array}$} & \multicolumn{2}{|c|}{$\begin{array}{l}\text { Partial effect } \\
\text { of } \mathrm{HL}\end{array}$} & \multicolumn{2}{|c|}{ Sobel's test } \\
\hline & $b$ & SE & $b$ & SE & $b$ & SE & $b$ & SE & $b$ & SE & $b$ & SE \\
\hline Non-verbal WM & -0.15 & 0.32 & $1.61^{*}$ & 0.53 & -0.06 & 0.51 & -0.96 & 0.58 & 0.40 & 0.40 & $-2.6 I^{*}$ & 0.28 \\
\hline Verbal WM & $-1.09^{*}$ & 0.36 & 0.56 & 0.65 & -0.26 & 0.62 & 0.30 & 0.71 & -0.95 & 0.49 & - & - \\
\hline Switching & 26.23 & $|8.6|$ & 24.83 & 32.15 & 42.65 & 30.96 & 55.71 & 35.03 & 15.29 & 24.33 & - & - \\
\hline Inhibition & -48.70 & 41.55 & -30.80 & 78.77 & -56.54 & 72.03 & 72.13 & 77.49 & -87.61 & 54.81 & - & - \\
\hline
\end{tabular}

$* p<.05$

$R^{2}=.12$, respectively). None of the other subscales had a significant effect on WM $(p>.05)$, neither did we find a significant relationship between any of the subscales and switching or working memory $(p>.05)$.

Subsequent regression analyses showed that perceived HL appreciation was an influence on the two bilingual groups' performance on both the verbal and the nonverbal WM tasks, but not in switching or inhibition (Table 3). The effect of perceived HL tolerance on nonverbal WM was medium $\left(R^{2}=.20\right)$. The Sobel test showed a statistically significant result, which indicates that differences in nonverbal WM between the two bilingual groups were mediated by perceptions of the teacher's HL tolerance. The mediation was only partial because initially there were no significant differences between the two bilingual groups in nonverbal WM, but there was an indirect relationship via perceived HL tolerance. For verbal WM, the significant differences between the two bilingual groups disappeared after including the subscales of appreciation as predictors, but none of these subscales was significantly associated with verbal WM. Therefore it was concluded that a teacher's appreciation of the HL did not function as mediator with respect to group differences in verbal WM.

\section{Discussion}

In this study, we investigated whether bilingual pupils with different home languages (HLs) differed in perceptions about the extent to which their teachers appreciate their HL, and whether these perceptions influence their executive functioning (EF). We studied EFs and perceptions of teachers' appreciation of Turkish- and German-speaking pupils in Dutch primary schools. We found that Turkish-Dutch children perceived less teacher appreciation of their HL than their German-Dutch peers, and these perceptions were related to nonverbal working memory (WM) performance. German bilinguals seem to profit more from their bilingualism than their Turkish-Dutch peers, and this appears to be partly related to teachers' appreciation of their HL. Our results show that teachers are of influence on bilingual children's cognitive outcomes, by providing classroom environments with varying degrees of appreciation of children's bilingualism.

Our findings - that bilingual children outperformed monolingual children on nonverbal WM and switching tasks - are in line with previous findings (Bialystok \& Viswanathan, 2009; Morales et al., 2013). Managing and switching between two languages is assumed to result in enhanced switching abilities (Green, 1998), as well as a more efficient processing of information in WM (Adesope et al., 2011). In contrast to findings from previous studies (Bialystok and 
Viswanathan, 2009; Morales et al., 2013), we did not find a bilingual advantage in inhibition or verbal WM. For inhibition, the task might have been too difficult (as the relatively high error rates of $9.0 \%$ indicate). However, our results are in line with those of Antón et al. (2014), who used a comparable task to assess inhibition in children of a similar age group. For verbal WM, our results are in line with those found by Luo, Craik, Moreno, \& Bialystok (2013), who additionally argue that due to a bilingual disadvantage in verbal tasks, the WM advantage is especially prone in the nonverbal domain. Furthermore, bilingual advantages are especially evident when the task includes additional EF demands (Morales et al., 2013). A possible explanation for our null findings could therefore be that the Digit Span relies only on verbal WM, and does not invoke other EF components.

As mentioned, we found that German-Dutch bilinguals perceived more appreciation of their HL than Turkish-Dutch bilinguals. This provides evidence for the fact that also in the sociolinguistic context of the classroom, some languages are valued less than others, and that this seems to be especially the case for languages spoken by ethnic minority groups (Extra \& Yağmur, 2004). It seems that, overall, the teachers in our sample behaved in line with governmental policies aimed at discouraging of the use of minority languages in schools (Kuiken \& Van der Linden, 2013).

The suggested benefits of being bilingual at school were confirmed by our results: that differential perceptions of teachers' appreciation of the HL, especially those of HL tolerance, were related to differences between the bilingual groups in the WM domain. The more positive children's perceptions of tolerance were, the better they performed on nonverbal WM tasks. An explanation for these findings may be that children who perceive that their teachers tolerate their HL more, are educated in an environment in which they have more opportunities to use their HL as well as Dutch. By consequence, they may feel more acknowledged as bilinguals. In such an environment, children may use and switch more between two languages. This requires ongoing inhibition of one language, and managing linguistic competition between words in two languages, which, according to the current theory on EF-development, may result in enhanced EFs. However, much about the influence of environmental contexts on EF still remains unknown. Future research should investigate how different educational contexts may influence EF, and what the additional influence of HL value on EF is. We could not find any relationship between perceptions of appreciation of the HL, and either switching or inhibition. Further research is needed to get more insight into the relationship between perceived appreciation and attentional control.

This study, in which we investigated the effect of sociolinguistic context features on bilingual advantages in EF, suffered from some limitations that prevent us from drawing particularly strong conclusions. First, we had a specific recruitment method in order to avoid dependency of the data, and restrictions concerning age range and specific home languages. Also, each child had to be tested individually, and we were dependent on participants' goodwill to participate in our study. Recruitment and data gathering were therefore very time consuming, and this resulted in relatively small groups with a limited variety in terms of socioeconomic backgrounds. Large-scale research, with more diverse groups with respect to SES, is needed to investigate how robust our results are.

As we focused on pupils' perceptions of teachers' appreciation of the home language, we did not observe the factual language use in classrooms. Future research could examine the extent to which children indeed use two languages and switch between two languages as predictors of EF benefits of bilinguals, for example, through observations in the classroom.

We showed once more that bilingual children show advantages in cognition over monolingual children. We also demonstrated, however, that it seems that these advantages cannot be dissociated from the sociolinguistic (classroom) context. Despite the abovementioned limitations and the exploratory nature of this research, the results therefore provide evidence for the hypothesis that the sociolinguistic context is of influence on bilingual advantages, at least in WM. 
Our results have some implications for policy and practice. In the Netherlands, nowadays, many schools follow a 'Dutch-only policy': they have implemented the school rule that in the school children should speak Dutch only (Frijns, 2014). This implies that children are frustrated in the practice of their bilingualism and, consequently, the development of the cognitive benefits of being bilingual is limited by these contexts. Whereas, according to the Dutch government, speaking another language would hinder integration (Kuiken \& Van der Linden, 2014), and lessen the use of Dutch, possibly resulting in a language delay in Dutch (Leseman, 2000), we showed that a sociolinguistic context in which languages are highly valued may also have positive consequences. Following the theoretical knowledge about stimulating EF, it seems that an educational context in which bilingualism is valued provides children with the opportunities to use and switch between two languages, which in turn could enhance their EFs. If future research revealed that this is a causal relationship, the government should reconsider restrictive policies regarding the use of languages of minority groups in schools.

In addition, teachers should be made aware of the benefits of bilingualism, and the consequences of their appreciation of different HLs for children's cognition. Teacher educators should play an important role in this process, since they could provide (prospective) teachers with the knowledge and skills that are needed to prepare them to be culturally responsive (Brown, 2007; Gay, 2002; Villegas \& Lucas, 2007).

This study also has implications for scientific research. We combined sociolinguistic theory with a cognitive-psychological approach to cognitive functioning. The results, showing that the cognitive-psychological outcomes of bilingual children can be better understood by taking into account a sociolinguistic research perspective, demonstrate the importance of a multidisciplinary research approach on the benefits of bilingualism.

\section{Funding}

The author(s) received no financial support for the research, authorship, and/or publication of this article.

\section{References}

Adesope, O. O., Lavin, T., Thompson, T., \& Ungerleider, C. (2010). A systematic review and meta-analysis of the cognitive correlates of bilingualism. Review of Educational Research, 80(2), 207-245.

Agirdag, O. (2010). Exploring bilingualism in a monolingual school system: Insights from Turkish and native pupils from Belgian schools. British Journal of Sociology of Education, 31(3), 207-321.

Agirdag, O., Jordens, K., \& Van Houtte, M. (2014). Speaking Turkish in Belgian primary schools: Teacher beliefs versus effective consequences. Bilig, 70(3), 7-28.

Antón, E., Duñabeitia, J. A., Estévez, A., Hernández, J. A., Castillo, A., Fuentes, L. J., .. Carreiras, M. (2014). Is there a bilingual advantage in the ANT task? Evidence from children. Frontiers in Psychology, 5(398), 1-12.

Appel, R., \& Muysken, P. (2005). Language contact and bilingualism. Amsterdam: Amsterdam University Press.

Atwill, K., Blanchard, J., Christie, J., Gorin, J. S., \& García, H. S. (2010). English-language learners: Implications of limited vocabulary for cross-language transfer of phonemic awareness with kindergartners. Journal of Hispanic Higher Education, 9(2), 104-129.

Barac, R., \& Bialystok, E. (2012). Bilingual effects on cognitive and linguistic development: Role of language, cultural background, and education. Child Development, 83(2), 413-422.

Baron, R. M., \& Kenny, D. A. (1986). The moderator-mediator variable distinction in social psychological research: Conceptual, strategic, and statistical considerations. Journal of Personality and Social Psychology, 52, 844-863.

Bialystok, E. (2009). Bilingualism: The good, the bad and the indifferent. Bilingualism: Language and Cognition, 12(1), 3-11. 
Bialystok, E., \& Martin, M. (2004). Attention and inhibition in bilingual children: Evidence from the Dimensional Change Card Sort task. Developmental Science, 7(3), 325-339.

Bialystok, E., \& Viswanathan, M. (2009). Components of executive control with advantages for bilingual children in two cultures. Cognition, 112(3), 494-500.

Blair, C., \& Peters Razza, R. (2007). Relating effortful control, executive function, and false belief understanding to emerging math and literacy ability in kindergarten. Child Development, 78(2), 647-663.

Brown, M. R. (2007). Educating all pupils: Creating culturally responsive teachers, classrooms and schools. Intervention in School and Clinic, 43, 57-62.

Carlson, S. M., \& Meltzoff, A. N. (2008). Bilingual experience and executive functioning in young children. Developmental Science, 11(2), 282-298.

Chun, H., \& Dickson, G. (2011). A psychoecological model of academic performance among Hispanic adolescents. Journal of Youth and Adolescence, 40(12), 1581-1594.

Cohen, J. (1988). Statistical power analysis for the behavioral sciences (2nd edn). Hillsdale, NJ: Lawrence Earlbaum Associates.

Cohen, J. (1992). A power primer. Psychological Bulletin, 112(1), 155-159.

Cronbach, L. J. (1951). Coefficient alpha and the internal structure of tests. Psychometrika, 16(3), 297-334.

Diamond, A. (2013). Executive functions. Annual Review of Psychology, 64, 135-168.

Diamond, A., Barnett, W. S., Thomas, J., \& Munro, S. (2007). Preschool program improves cognitive control, Science, 318, 1387-1388.

Eisenchlas, S. A., Schalley, A. C., \& Guillemin, D. (2013). The importance of literacy in the home language. SAGE Open, 3(4), 1-14.

Extra, G., \& Yağmur, K. (2004). Meertaligheid in multicultureel Europa: De status van immigrantentalen thuis en op school. Levende Talen Tijdschrift, 5(1), 13-18.

Frijns, C. (2014). Ont-armen of omarmen? Over onze omgang met (meer) talen op school. Meer Taal, 3(1), $5-7$.

Garon, N., Bryson, S. E., \& Smith, I. M. (2008). Executive function in preschoolers: A review using an integrative framework. Psychological Bulletin, 134(1), 31-60.

Gay, G. (2002). Preparing for culturally responsive teaching. Journal of Teacher Education, 53, 106-116.

Gkaintartzi, A., \& Tsokalidou, R. (2011). "She is a very good child but she doesn't speak": The invisibility of children's bilingualism and teacher ideology. Journal of Pragmatics, 43, 588-601.

Green, D. W. (1998). Mental control of the bilingual lexico-semantic system. Bilingualism: Language and Cognition, 1, 67-81.

Hackman, D. A., \& Farah, M. J. (2009). Socioeconomic status and the developing brain. Trends in Cognitive Sciences, 13(2), 65-73.

Kort, W., Schittekatte, M., Dekker, P. H., Verhaeghe, P., Compaan, E. L., Bosmans, M., \& Vermeir, G. (2005). WISC-III NL Wechsler Intelligence Scale for Children. Derde Editie NL. Handleiding en Verantwoording. Amsterdam: Harcourt Test Publishers/Nederlands Instituut voor Psychologen.

Kuiken, F., \& Van der Linden, E. (2014). Language policy and language education in the Netherlands and Romania. Dutch Journal of Applied Linguistics, 2(2), 205-223.

Lee, J. S., \& Oxelson, E. (2006). "It's not my job": K-12 teacher attitudes toward pupil's heritage language maintenance. Bilingual Research Journal: The Journal of the National Association for Bilingual Education, 30(2), 453-477.

Leseman, P. P. M. (2000). Bilingual vocabulary development of Turkish preschoolers in the Netherlands. Journal of Multilingual and Multicultural Development, 21(2), 93-112.

Luk, G., De Sa, E., \& Bialystok, E. (2011). Is there a relation between onset age of bilingualism and enhancement of cognitive control? Bilingualism: Language and Cognition, 14(4), 588-595.

Luo, L., Craik, F. I. M., Moreno, S., \& Bialystok, E. (2013). Bilingualism interacts with domain in a WM task: Evidence from aging. Psychology and Aging, 28(1), 28-34.

Miyake, A., Friedman, N. P., Emerson, M. J., Witzki, A. H., Howerter, A., \& Wager, T. D. (2000). The unity and diversity of executive functions and their contributions to complex "frontal lobe" tasks: A latent variable analysis. Cognitive psychology, 41, 49-100. 
Moffitt, T. E., Arseneault, L., Belsky, D., Dickson, N., Hancox, R. J., Harrington, H., . .Caspi, A. (2011). A gradient of childhood self-control predicts health, wealth, and public safety. Proceedings of the National Academy of Sciences, 108(7), 2693-2698.

Morales, J., Calvo, A., \& Bialystok, E. (2013). Working memory development in monolingual and bilingual children. Journal of Experimental Child Psychology, 114(2), 187-202.

Raven, J. C., Court, J. H., \& Raven, J. C. (1990). Manual for Raven's Progressive Matrices and Vocabulary Scales - section 2: Coloured Progressive Matrices. Oxford: Oxford Psychology Press.

Sobel, M. E. (1982). Asymptotic confidence intervals for indirect effects in structural equation models. Sociological Methodology, 13, 290-312.

Soveri, A., Rodriguez-Fornells, A., \& Laine, M. (2011). Is there a relationship between language switching and executive functions in bilingualism? Introducing a within-group analysis approach. Frontiers in Psychology, 2, 138-143.

St Clair-Thompson, H. L., \& Gathercole, S. E. (2006). Executive functions and achievements in school: Shifting, updating, inhibition, and working memory. The Quarterly Journal of Experimental Psychology, 59(4), 745-759.

Vandierendonck, A., Kemps, E., Fastame, M. C., \& Szmalec, A. (2004). Working memory components of the Corsi blocks task. British Journal of Psychology, 95(1), 57-79.

Van Gaalen, R., \& De Vos, A. (2011). Sociaaleconomische positie van ouders en kinderen naar herkomst. Bevolkingstrends, 22-27.

Villegas, A. M., \& Lucas, T. (2007). The culturally responsive teacher. Educational Leadership, 64(6), 28-33.

\section{Author biographies}

Claire Goriot is a PhD student at the Centre for Language Studies of Radboud University, Nijmegen, The Netherlands. Her research interests include bilingualism, linguistic and cognitive development (especially executive functioning) of bilinguals, and (bilingual) education.

Eddie Denessen is an associate professor at the Behavioural Science Institute, Radboud University Nijmegen. He has a background in sociology of education and educational research methods. His research interests include educational inequality, school family partnerships, and teacher expectations and classroom differentiation.

Joep Bakker, $\mathrm{PhD}$, is an assistant professor in the departments of Special Education and Educational Sciences at Radboud University, Nijmegen, The Netherlands, and a member of the Behavioural Science Institute. His research and teaching activities focus i.e. on inclusive education, school-parent-relationships, interethnic attitudes and relations of students in elementary education.

Mienke Droop is an assistant professor of Educational Sciences at the Behavioural Science Institute, Radboud University in Nijmegen. Her research focuses on language and literacy development in first- and second-language learners and literacy instruction and interventions in primary education. 